FIORESE, D. A.; MARASCA, I.; FERNANDES, B.B.; SANDI, J.; MORELLI-FERREIRA, F.; LANÇAS, K. P. Desempenho de três tratores agrícolas em ensaios de tração. Revista de Agricultura Neotropical, Cassilândia-MS, v. 2, n. 2, p. 68-76, abr./jun. 2015.

\title{
DESEMPENHO DE TRÊS TRATORES AGRÍCOLAS EM ENSAIOS DE TRAÇÃO
}

\section{DIEGO AUGUSTO FIORESE ${ }^{1}$, INDIAMARA MARASCA ${ }^{2}$, BÁRBARA BARRETO FERNANDES ${ }^{2}$, JEFFERSON SANDI $^{2}$, FRANCIELLE MORELLI-FERREIRA ${ }^{3}$, KLÉBER PEREIRA LANÇAS ${ }^{2}$}

\footnotetext{
${ }^{1}$ Universidade Federal de Mato Grosso-UFMT, Sinop/MT, Brasil, dafiorese@yahoo.com.br; ${ }^{2}$ Faculdade de Ciências Agronômicas da Universidade Estadual Paulista Júlio de Mesquita Filho-FCA/UNESP, Departamento de Engenharia Rural, Botucatu/SP, Brasil, marasca_7@hotmail.com, babarretof@hotmail.com, jeffe_sandi@hotmail.com, kplancas@fca.unesp.br;

${ }^{3}$ Universidade do Estado de Mato Grosso-UNEMAT, Alta Floresta/MT, Brasil, fran_morelli_@hotmail.com
}

RESUMO: A avaliação de desempenho de tratores agrícolas, levando-se em consideração a condição do solo, é importante para identificar a máxima capacidade de tração e o consumo de combustível de acordo com os níveis de esforços exigidos. Neste sentido, o objetivo do trabalho foi realizar ensaios de tração para determinação do desempenho de tratores agrícolas sobre pista de solo firme sem cobertura vegetal. Foram avaliados três modelos de tratores $4 \times 2$ TDA, e seis níveis de esforços (força de tração) na barra de tração $(5,10,15,20,25$ e $27 \mathrm{kN}$ ). Para o fator trator, as médias foram comparadas pelo teste de Tukey, e para o fator força de tração, as médias foram ajustadas por equações de regressão. Observou-se que o menor desempenho energético representado pelo consumo de combustível, foi obtido com o trator que operava com o maior regime de rotação. Houve comportamento linear crescente em função da força de tração, para os parâmetros consumo/horário, potência e eficiência na barra de tração e ajuste não linear para patinagem e consumo específico.

PALAVRAS-CHAVE: Consumo, interação solo-máquina, potência, ensaio.

\section{PERFORMANCE OF THREE AGRICULTURAL TRACTORS IN DRAWBAR TEST}

\begin{abstract}
The performance evaluation of agricultural tractors, according on the soil condition, it is important to identify the maximum drawbar pull and fuel consumption according to the level of effort required. The objective was to perform drawbar tests to determine the performance of agricultural tractors on firm soil. Three models of tractors MFWD, and six levels of effort (drawbar pull) on the drawbar $(5,10,15,20,25$ and $27 \mathrm{kN})$ were evaluated. For tractor factor, the means were compared by Tukey test, and the drawbar pull factor, the means were adjusted by regression equations. The lower energy performance was obtained with the tractor that operated with higher engine speed. There was an increasing linear behavior as a function of traction force to the parameters hourly consumption, power and efficiency to the drawbar and nonlinear fit for skating and specific fuel consumption.
\end{abstract}

KEYWORDS: Consumption, soil machine interaction, power, test.

\section{INTRODUÇÃO}

Os solos agrícolas podem apresentar inúmeras condições superficiais e subsuperficiais, no que diz respeito à textura, adensamento, tipo de cobertura, teor de água, entre outros. Estas condições são refletidas na mecanização agrícola, em especial na interação solo-máquina, onde os efeitos podem ser negativos para o desempenho de tratores, 
principalmente em casos de solos agrícolas, que oferecem pouca aderência para os rodados motrizes.

Nas últimas décadas, a mecanização das operações agrícolas tem se intensificado resultando em aumento na carga aplicada ao solo pelas máquinas agrícolas e, consequentemente, em modificações nas propriedades físicas do solo (YAVUZCAN et al., 2005). Para Horn e Lebert (1994), a aplicação de cargas dinâmicas por rodados e implementos agrícolas no solo produz tensões na interface solo-pneu e solo-implemento, tanto em superfície quanto em profundidade as quais afetam na compactação do solo e também no desenvolvimento de tração.

De acordo com Gabriel Filho et al. (2010), o desempenho na barra de tração (BT) de um trator agrícola, depende de diversos fatores destacando a distribuição de peso sobre os rodados e a condição da superfície do solo. Em complemento, Yanai et al. (1999), ressaltam que a eficiência de tração pode variar em função de algumas propriedades do solo, como a textura e umidade, além da cobertura existente.

Um trator operando sobre superfície de solo firme, segundo a norma D497.7 da "American Society of Agricultural and Biological Engineers" (ASABE, 2011), apresenta um desempenho na barra de tração com eficiência máxima de aproximadamente 68\%, considerada intermediária se comparada com pista padrão de concreto (maior eficiência) e com solos cultivados e mobilizados (menor eficiência).

Gabriel Filho et al. (2004), realizaram trabalhos de tração em solos agrícolas e concluíram que os índices de patinagem são menores com consequente aumento da eficiência de tração para condições superficiais onde se tenha menor quantidade de matéria seca. Trabalhos realizados por Serrano (2007), com diversos modelos de tratores e em distintas condições de tração, mostraram um comportamento linear crescente do consumo de combustível em função do aumento da força de tração por unidade de largura de trabalho.

Zoz e Grisso (2003), analisando parâmetros relacionados com a teoria da tração, obtiveram gráficos de patinagem com comportamento exponencial em função da carga na barra de tração do trator, reduzindo a eficiência trativa para condições de alta patinagem.

De acordo com Lopes et al. (2003), o consumo específico de combustível é a maneira mais técnica de expressar o gasto de combustível pode ser utilizada para comparar motores, tratores e equipamentos de tamanho e formas diferentes, pelo fato de considerar a massa e a potência produzida. Márquez (2012) acrescenta que o consumo específico de combustível, em função da demanda de tração, não possui comportamento linear e pode ser afetado pela patinagem dos rodados motrizes.

Os motores Diesel apresentam maior eficiência em baixas rotações, em função da maior capacidade em admitir oxigênio (comburente), maior tempo para completar o ciclo da combustão e em função do menor atrito entre os componentes dinâmicos e estáticos do motor (MÁRQUEZ, 2012). Neste sentido, motores que operam em menores regimes de rotação tem grande tendência à um menor consumo de combustível.

Considerando a importância de se comparar diferentes tratores em função de suas peculiaridades construtivas e seu desempenho em função da variação do nível de esforço, este estudo consistiu em avaliar o desempenho de três tratores agrícolas distintos, submetidos a ensaios de tração sobre uma pista de solo firme sem cobertura vegetal. 


\section{MATERIAL E MÉTODOS}

O trabalho foi conduzido no Núcleo de Ensaios de Máquinas e Pneus Agroflorestais (NEMPA), situado na Faculdade de Ciências Agronômicas da Universidade Estadual Paulista, FCA/UNESP com localização geográfica nas coordenadas $22^{\circ} 50^{\prime} 21.04 " \mathrm{~S}$ e $48^{\circ} 25^{\prime} 19.83^{\prime \prime} \mathrm{O}$. Quanto à caracterização relacionada à interação solo-máquina e para a trafegabilidade de máquinas agrícolas a área possuía uma estrutura superficial e subsuperficial denominada como pista de solo firme (solo batido), com adensamento que permite pouca deformação do solo, e bom rendimento de tração. A textura é argilosa com teor de argila superior a $50 \%$, classificado como Nitossolo Vermelho Distroférrico, conforme o sistema brasileiro de classificação de solos (EMBRAPA, 1999). Na camada superficial onde ocorre a interação solo-máquina o solo estava com teor de água de $8,5 \%$; devido a este índice e compactação não foi possível determinar a resistência à penetração com penetrômetro manual. Desta maneira, estima-se um índice de cone superior a $10 \mathrm{MPa}$, em toda a extensão da área (400 x $30 \mathrm{~m}$ ) onde foram montadas as parcelas.

No experimento adotou-se o delineamento experimental em blocos ao acaso (DBC), onde foram avaliados os seguintes tratamentos: três tratores agrícolas novos, munidos de tração dianteira auxiliar (TDA), com potências de catálogo do motor (fornecidas pelo fabricante) de 69,9; 69,9 e 78,7 kW; e seis níveis de esforços na barra de tração (BT), sendo: $5,10,15,20,25$ e $27 \mathrm{kN}$. Para cada tratamento foram realizadas três repetições em parcelas de $30 \mathrm{~m}$ de comprimento e largura de $2,5 \mathrm{~m}$, totalizando 54 unidades experimentais. Para cada trator utilizou-se apenas uma marcha de trabalho a qual proporcionou as velocidades mais próximas entre ambos (entre 6 e $7 \mathrm{~km} \mathrm{~h}^{-1}$ ), sendo ainda uma das marchas mais usuais para as diversas operações agrícolas. As especificações principais dos três tratores estão descritas na Tabela 1.

Tabela 1. Principais especificações dos tratores utilizados nos ensaios de tração

\begin{tabular}{|c|c|c|c|}
\hline Trator & $\mathrm{T} 1$ & $\mathrm{~T} 2$ & $\mathrm{~T} 3$ \\
\hline Classificação & \multicolumn{3}{|c|}{ Monobloco $-4 \times 2 \mathrm{TDA}^{\mathrm{a}}$} \\
\hline Motor & \multicolumn{3}{|c|}{4 cilindros, sobrealimentado (turbo) } \\
\hline Potência de catálogo do motor (kW) & 69,9 & 69,9 & 78,7 \\
\hline Rotação máxima do motor (rpm) & 2330 & 2700 & 2270 \\
\hline Rotação nominal (rpm) & 2200 & 2500 & 2100 \\
\hline Massa total lastrado $(\mathrm{kN})$ & 52,2 & 55,2 & 56,6 \\
\hline Distribuição de massa nos eixos (\%) & $\begin{array}{l}60 \% \text { traseiro } \\
40 \% \text { dianteiro }\end{array}$ & $\begin{array}{l}57 \% \text { traseiro } \\
43 \% \text { dianteiro }\end{array}$ & $\begin{array}{l}59 \% \text { traseiro } \\
41 \% \text { dianteiro }\end{array}$ \\
\hline Pneus traseiros (pressão, kPa) & \multicolumn{3}{|l|}{$18.4-34(124,2)$} \\
\hline Pneus dianteiros (pressão, kPa) & \multicolumn{3}{|l|}{$14.9-24(138,0)$} \\
\hline
\end{tabular}

TDA ${ }^{\mathrm{a}}$ - Tração dianteira assistida.

Os níveis de esforços na BT foram aplicados com a Unidade Móvel de Ensaio na Barra de Tração (UMEB), descrita por Gabriel Filho et al. (2008), munida de seis rodados com freios a tambor e com controle pneumático variável de frenagem (Figura 1).

Nos ensaios de tração, o procedimento consistiu em realizar frenagens controladas na BT, iniciando-se com o trator em movimento (até estabilização da velocidade) e com máxima rotação do motor, até a atingir a carga desejada. Utilizaram-se os seguintes equipamentos eletrônicos embarcados no conjunto trator-UMEB para obtenção dos dados de interesse: célula de carga Líder 10 t, capacidade máxima de $100 \mathrm{kN}$, para verificação da carga na BT; dois fluxômetros modelo OVAL M-III LSF41L0-M2, para determinação do consumo de 
combustível; cinco sensores de rotação tipo encoder rotativo modelo S\&E E2A1A 24V com 60 pulsos por volta, para determinação da patinagem dos rodados e para velocidade real junto a uma roda odométrica; encoder rotativo modelo S\&E E2A1A 24V com dois pulsos por volta acoplado ao eixo da TDP para determinação da rotação do motor; sensores de temperatura tipo pt100 para monitoramento e obtenção da temperatura do combustível, da água do radiador e do óleo do motor; e sistema de aquisição de dados tipo CLP 24V modelo Vision $230^{\mathrm{TM}}$, para captação e armazenamento dos dados.

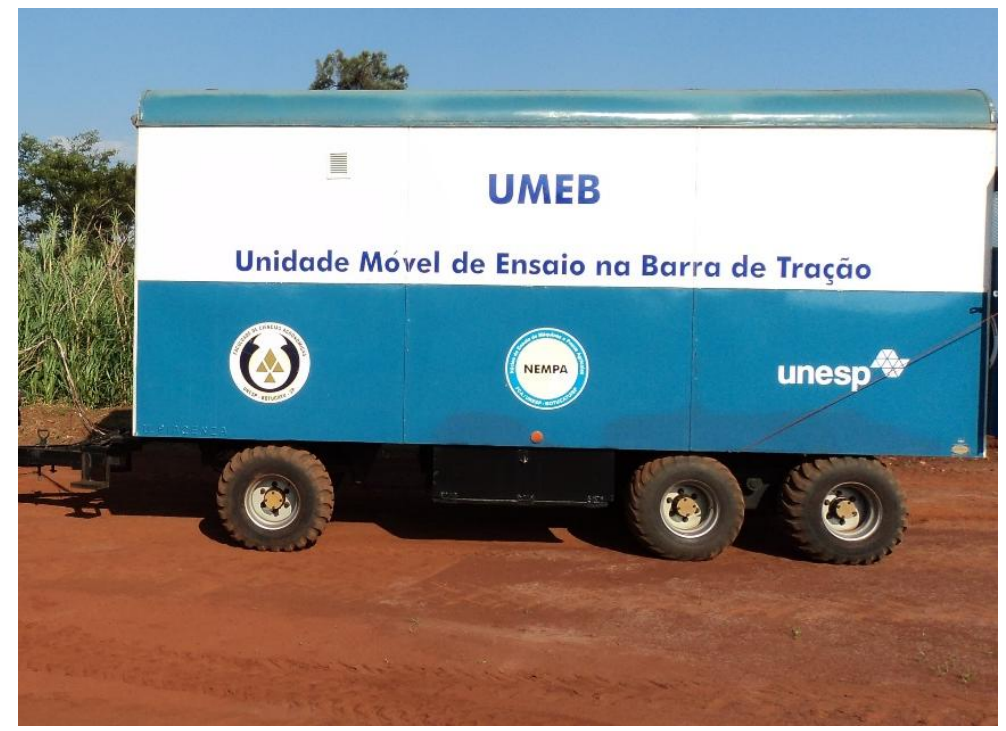

Figura 1. Vista lateral da UMEB com seis rodados para frenagem e sobre a pista de solo firme. (Fonte: Diego Augusto Fiorese, 2012).

A velocidade real e a força de tração foram obtidas diretamente pelo sistema de aquisição de dados. Para a determinação da rotação do motor, foi utilizada a equação 1.

$\mathrm{n}=\left(\left(\frac{\mathrm{Pul}_{\mathrm{TDP}}}{2}\right) \cdot \mathrm{i}\right) /\left(\frac{\mathrm{t}}{60}\right)$

onde:

$\mathrm{n}$ - rotação do motor $(\mathrm{rpm})$

$\mathrm{Pul}_{\mathrm{TDP}}$ - número de pulsos obtidos na TDP durante o tempo de coleta na parcela

i - relação de transmissão entre motor e TDP

$\mathrm{t}$ - tempo de coleta de dados na parcela de $30 \mathrm{~m}(\mathrm{~s})$

60 - constante para conversão em minutos

Os principais parâmetros avaliados, foram: patinagem dos rodados motrizes (Pat), consumo horário volumétrico (Chv), consumo específico de combustível $(\mathrm{Ce})$, potência na barra de tração $\left(\mathrm{P}_{\mathrm{BT}}\right)$, eficiência na barra de tração (Ef. BT). O Chv foi obtido diretamente pelo sistema de aquisição de dados. Para os demais parâmetros, as equações utilizadas são descritas a seguir.

Pat $=\frac{\mathrm{N}_{1}-\mathrm{N}_{0}}{\mathrm{~N}_{1}} \cdot 100$ 
em que:

Pat - patinagem (\%)

$\mathrm{N}_{0}$ - número de pulsos nas rodas motrizes sem carga na parcela de $30 \mathrm{~m}$

$\mathrm{N}_{1}$ - número de pulsos nas rodas motrizes com carga, na parcela de $30 \mathrm{~m}$

$\mathrm{Ce}=\frac{\rho \cdot \mathrm{Chv}}{\mathrm{P}_{\mathrm{BT}}}$

em que,

Ce - consumo específico $\left(\mathrm{g} \mathrm{kWh}^{-1}\right)$;

$\rho$ - densidade do combustível em determinada temperatura $\left(\mathrm{g} \mathrm{L}^{-1}\right)$

$\mathrm{P}_{\mathrm{BT}}$ - potência na barra de tração $(\mathrm{kW})$

$\mathrm{P}_{\mathrm{BT}}=\mathrm{FT} . \mathrm{V}$

Onde:

$\mathrm{P}_{\mathrm{BT}}$ - Já descrito anteriormente

FT - força de tração $(\mathrm{kN})$

$\mathrm{V}$ - velocidade real $\left(\mathrm{m} \mathrm{s}^{-1}\right)$

Ef. $\mathrm{BT}=\frac{\mathrm{P}_{\text {cat }}}{\mathrm{P}_{\mathrm{BT}}}$

Onde:

Ef. BT - Eficiência na barra de tração (\%)

$\mathrm{P}_{\text {cat }}$ - Potência de catálogo fornecida pelo fabricante do trator $(\mathrm{kW})$

$\mathrm{P}_{\mathrm{BT}}-$ Já descrito anteriormente

Os dados foram submetidos à análise de variância, sendo que os níveis do fator qualitativo (tratores) foram comparados pelo teste de Tukey, ao nível de 5\% de probabilidade, enquanto que para os níveis do fator quantitativo (força de tração), procedeu-se o ajustamento com modelos de regressão.

\section{RESULTADOS E DISCUSSÃO}

Para comparação entre os tratores, na Tabela 2 apresenta-se os resultados médios obtidos para a carga na BT, para a velocidade real de deslocamento e para a rotação final. A força de tração média ficou próxima para os três modelos, e assim permite a comparação entre ambos, considerando que houve pouca variação do nível de esforço. Quanto a velocidade média, verifica-se que os modelos resultaram em valores diferentes, mas aproximados. A comparação é válida, tendo em vista que em uma operação agrícola a seleção da marcha é feita da mesma forma, selecionando-se a relação de transmissão mais aproximada entre ambos, ou a mais próxima da velocidade que se deseja (por exemplo 6,5 $\mathrm{km} \mathrm{h}^{-1}$ ). 
Tabela 2. Resultados médios obtidos da força de tração, velocidade e rotação do motor, para os três tratores avaliados

\begin{tabular}{cccc}
\hline Trator & $\begin{array}{c}\text { Força de tração } \\
\text { média }(\mathrm{kN})\end{array}$ & $\begin{array}{c}\text { Velocidade média } \\
\text { final }\left(\mathrm{km} \mathrm{h}^{-1}\right)\end{array}$ & $\begin{array}{c}\text { Rotação média } \\
\text { do motor }(\mathrm{rpm})\end{array}$ \\
\hline $\mathrm{T} 1$ & 14,7 & 6,4 & 2265 \\
$\mathrm{~T} 2$ & 14,8 & 6,5 & 2600 \\
$\mathrm{~T} 3$ & 14,8 & 6,7 & 2170 \\
\hline
\end{tabular}

Na Tabela 3 pode-se observar os resultados da análise de variância para o fator trator, para cinco parâmetros de desempenho. Para o consumo horário volumétrico (Chv), verificouse diferença significativa entre os três tratores, sendo o T1 com menor valor $\left(13,8 \mathrm{~L} \mathrm{~h}^{-1}\right)$ e o T2 o maior valor $\left(15,8 \mathrm{~L} \mathrm{~h}^{-1}\right)$. Já para o consumo específico (Ce), o trator 2 (T2) diferiu estatisticamente dos demais, tendo o maior valor de $520 \mathrm{~g} \mathrm{kWh}^{-1}$. Conforme Lopes et al. (2003), o consumo específico é um parâmetro que permite a comparação de tratores distintos e quanto maior o valor, menor é a eficiência da conversão energética do combustível (desvantagem para o T2).

Quanto aos parâmetros potência na barra de tração $\left(\mathrm{P}_{\mathrm{BT}}\right)$ e eficiência na barra de tração (Ef. BT), também houve diferença entre os três modelos, tendo-se o T2 com maior valor médio de eficiência na BT $(41,4 \%)$. Para o parâmetro patinagem, não houve diferença entre os modelos avaliados, tendo-se uma média de 6,4\%. Gabriel Filho et al. (2010), em ensaios sobre pista de solo firme, obtiveram resultados similares.

Tabela 3. Resultados médios da análise de variância para o fator trator para os parâmetros consumo horário volumétrico (Chv), consumo específico $(\mathrm{Ce})$, potência na barra de tração (PBT), eficiência na barra de tração (Ef. BT) e patinagem (Pat).

\begin{tabular}{cccccc}
\hline Trator & $\begin{array}{c}\text { Pat } \\
(\%)\end{array}$ & $\begin{array}{c}\mathrm{Chv} \\
\left(\mathrm{L} \mathrm{h}^{-1}\right)\end{array}$ & $\begin{array}{c}\mathrm{Ce} \\
\left(\mathrm{g} \mathrm{kWh}^{-1}\right)\end{array}$ & $\begin{array}{c}\mathrm{P}_{\mathrm{BT}} \\
(\mathrm{kW})\end{array}$ & $\begin{array}{c}\text { Ef. BT } \\
(\%)\end{array}$ \\
\hline T1 & $6,6 \mathrm{a}$ & $13,8 \mathrm{c}$ & $470 \mathrm{~b}$ & $28,3 \mathrm{c}$ & $40,5 \mathrm{~b}$ \\
T2 & $6,5 \mathrm{a}$ & $15,8 \mathrm{a}$ & $520 \mathrm{a}$ & $28,9 \mathrm{~b}$ & $41,4 \mathrm{a}$ \\
T3 & $6,3 \mathrm{a}$ & $14,7 \mathrm{~b}$ & $485 \mathrm{~b}$ & $29,8 \mathrm{a}$ & $37,9 \mathrm{c}$ \\
\hline Média & 6,4 & 14,8 & 492 & 29,0 & 39,9 \\
DMS & 0,28 & 0,54 & 16,1 & 0,54 & 0,82 \\
C.V. $(\%)$ & 5,29 & 4,51 & 4,07 & 2,30 & 2,55 \\
\hline
\end{tabular}

Médias com letras iguais na coluna não diferem entre si pelo teste de Tukey $(P>0,05)$. C.V. - coeficiente de variação.

A análise do fator quantitativo - força de tração - foi analisada por regressões e são apresentadas na Figura 2. O comportamento da patinagem foi crescente com ajuste polinomial cubico (de quarta ordem). As curvas obtidas foram semelhantes com dados de Márquez (2012). Zoz e Grisso (2003), obtiveram resultados com ajuste exponencial da patinagem em função da força de tração, entretanto, o comportamento das curvas foi semelhante, podendo ser observado que há uma tendência do aumento mais acentuado da patinagem nas cargas acima de $20 \mathrm{kN}$. 

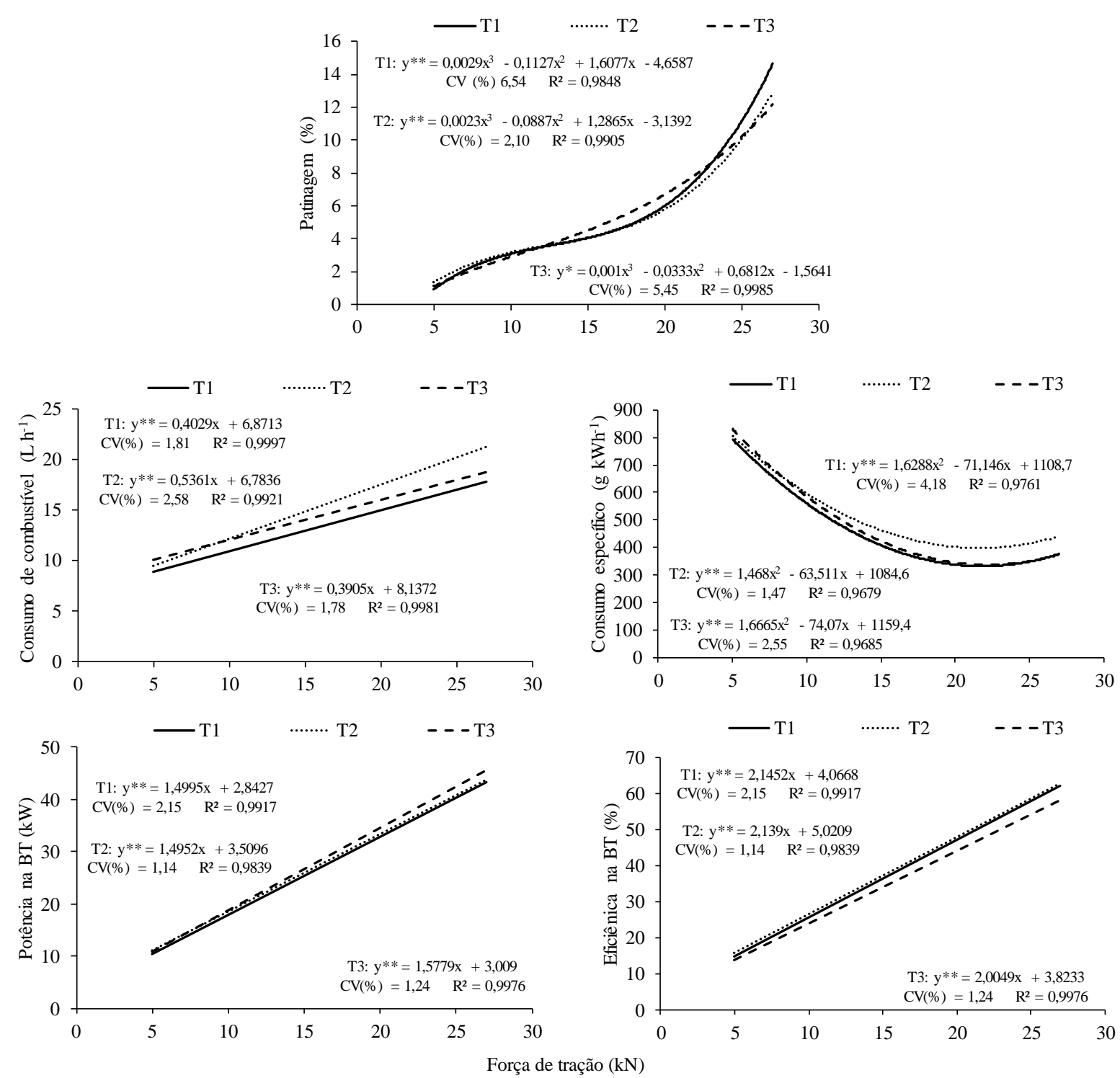

Figura 2. Regressões para patinagem, consumo horário de combustível, consumo específico, potência na barra de tração e eficiência na barra de tração, em função da força de tração.

Em geral, nos motores agrícolas, o maior consumo de combustível é atingido próximo a rotação de potência máxima e com o acelerador totalmente acionado, tendendo a reduzir o consumo com a queda de rotação do motor. Para as condições do solo e a velocidade adotada, o Chv apresentou ascendência linear de acordo com o aumento da força de tração, similar com resultados publicados por Serrano (2007). Já o consumo específico teve comportamento quadrático e decrescente com os menores resultados obtidos nas cargas entre 20 e $25 \mathrm{kN}$. Para os dois parâmetros de consumo (volumétrico e específico), o T2 apresentou os piores resultados. De acordo com as especificações dos modelos, observa-se que o T2 opera em um regime de rotação maior quando comparado com o T1 e T3, tendo sua rotação máxima livre de $2700 \mathrm{rpm}$ e a média final de $2600 \mathrm{rpm}$. Conforme relata Márquez (2012), maiores rotações são menos eficientes, tendo em vista que há um maior gasto energético em função do maior atrito entre os componentes móveis e estáticos do motor e também devido ao menor tempo para a combustão do combustível.

A potência e a eficiência na BT dos três tratores tiveram comportamento linear, explicado devido ao coeficiente de tração máximo ter sido abaixo do esperado. Em condições 
de maior capacidade trativa e para velocidades maiores, a potência e eficiência na BT tem comportamento não linear, considerando que há uma maior perda na patinagem dos rodados motrizes. A máxima eficiência na BT para os três tratores (próximo de 60\%) foi inferior ao valor médio sugerido pela ASABE (2011) que é de $68 \%$.

\section{CONCLUSÕES}

O trator 2 apresentou a melhor eficiência na barra de tração, no entanto, considerando que os parâmetros de desempenho energético são os mais úteis para avaliação de tratores agrícolas conclui-se que o trator 2 o qual operou com maior regime de rotação do motor, obteve a menor eficiência energética, tendo em vista que apresentou os maiores consumos de combustível dentre os três modelos avaliados.

A interação solo-máquina para a velocidade utilizada em cada um dos tratores, não permitiu que os parâmetros energéticos e de desempenho atingissem o máximo rendimento para a pista de solo firme.

\section{AGRADECIMENTOS}

Pelo apoio concedido a realização dos trabalhos, os autores agradecem ao Núcleo de Ensaios de Máquinas e Pneus Agroflorestais - NEMPA, e ao programa de Pós-Graduação em Energia na Agricultura, ambos integrados a Faculdade de Ciências Agronômicas da Universidade Estadual Paulista Júlio de Mesquita Filho, Botucatu-SP.

\section{REFERÊNCIAS BIBLIOGRÁFICAS}

ASABE. AMERICAN SOCIETY OF AGRICULTURAL AND BIOLOGICAL ENGINEERS. ASAE D497.7 Agricultural machinery management data. St. Joseph: ASABE Standards, 2011.8 p.

EMBRAPA. EMPRESA BRASILEIRA DE PESQUISA AGROPECUÁRIA. Centro Nacional de Pesquisa de Solos. Sistema brasileiro de classificação de solos. Rio de Janeiro: Embrapa, 1999, 412 p.

GABRIEL FILHO, A.; SILVA, S. L.; MODOLO, A. J.; SILVEIRA, J. C. M. Desempenho de um trator operando em solo com diferentes tipos de cobertura vegetal. Engenharia Agrícola. Jaboticabal-SP, v. 24, n. 3, p. 781-789, 2004.

GABRIEL FILHO, A.; LANÇAS, K. P.; GUERRA, S. P.; PAULA, C. A.; MONTEIRO, L. A. UMEB - Unidade Móvel Para Ensaio da Barra de Tração. Engenharia Agrícola, Jaboticabal-SP, v. 28, n. 4, p. 782-789, 2008.

GABRIEL FILHO, A.; MONTEIRO, L. A.; LANÇAS, K. P.; GUERRA, S. P. S.; JESUINO, P. R. Influência da altura das garras dos pneus de um trator em área de plantio direto. Revista Brasileira de Engenharia Agrícola e Ambiental. Campina Grande-PB, v. 14, n. 10, p. 1123-1128, 2010.

HORN, R.; LEBERT, M. Soil compactability and compressibility. In: SOANE, B.D.; OUWERKERK, C. Van. Soil compaction in crop production, Developments in Agricultural Engineering. Amsterdam: Elsevier, 1994, p. 45-69.

LOPES, A.; LANÇAS, K. P.; FURLANI, C. A.; NAGAOKA, A. K.; CASTRO NETO, P. C.; GROTTA, D. C. Consumo de combustível de um trator em função do tipo de pneu, da 
lastragem e da velocidade de trabalho. Revista Brasileira de Engenharia Agrícola e Ambiental, Campina Grande-PB, v. 7, n. 2, p. 382-386, 2003.

MÁRQUEZ, L. Tractores Agrícolas: Tecnologias y utilización. Madrid: B\&h Editores, 2012. $844 \mathrm{p}$.

SERRANO, J. M. P. R. Desempenho de tratores agrícolas em tração. Pesquisa Agropecuária Brasileira, Brasília-DF, v. 42, n. 7, p. 1021-1027, 2007.

YANAI, K.; SILVEIRA, G. M.; LANÇAS, K. P.; CORRÊA. I. M.; MAZIERO, J. V. G. Desempenho operacional de trator com e sem acionamento da tração dianteira auxiliar. Pesquisa Agropecuária Brasileira, Brasilia-DF, v. 34, n. 8, p. 1427-1434, 1999.

YAVUZCAN, H. G.; MATTHIES, D.; AUERNHAMMER, H. Vulnerability of Bavarian silty loam soil to compaction under heavy whell traffic: impacts of tillage method and soil water content. Soil and Tillage Research, Amsterdam, v. 84, n. 2, p.200-215, 2005.

ZOZ, F. M.; GRISSO, R. D. Traction and Tractor Performance. Louisville: ASAE, 2003. $46 \mathrm{p}$. 